\title{
Psicooncología
}

ISSN: 1696-7240

\section{Programa E-Health ICOnnecta't: un ecosistema para fomentar el bien- estar en cáncer a través de la propuesta europea ONCOMMUN}

Cristian Ochoa-Arnedo ${ }^{*}$; Aida Flix-Valle ${ }^{2}$; Joan Carles Medina ${ }^{3}$; Elisenda Escriche ${ }^{4}$; Ana Rodríguez $^{5}$; Claudia Villanueva ${ }^{6}$, Enric C. Sumalla ${ }^{7}$; Miquel Alabèrnia-Segura ${ }^{8}$; Claudia Prats ; María José Corral ${ }^{10}$; David Gallardo-Pujol ${ }^{11}$; Guillem Feixas ${ }^{12}$; Candela Calle ${ }^{13}$; Jordi Trelis ${ }^{14}$; Josep Maria Borràs ${ }^{15}$

1 Cristian Ochoa Arnedo. Programa E-Health ICOnnecta’t, Institut Català d'Oncologia. Dpto. Psicología Clínica y Psicobiología, Universitat de Barcelona. Institut d'Investigació Biomèdica de Bellvitge (IDIBELL) Barcelona, España.

E-mail: cochoa@iconcologia.net

2 Aida Flix-Valle. Programa E-Health ICOnnecta’t, Institut Català d'Oncologia. Dpto. Psicología Clínica y Psicobiología, Universitat de Barcelona. Institut d'Investigació Biomèdica de Bellvitge (IDIBELL) Barcelona, España. E-mail: aflixv@iconcologia.net

3 Joan Carles Medina. Institut d'Investigació Biomèdica de Bellvitge (IDIBELL). Dpto. Psicología i Ciencias de la Educación, Universitat Oberta de Catalunya, Barcelona, España

E-mail: jmedina1@uoc.edu

4 Elisenda Escriche. Programa E-Health ICOnnecta't, Institut Català d'Oncologia. Barcelona, España E-mail: eescriche@iconcologia.net

5 Ana Rodríguez. Programa E-Health ICOnnecta’t, Institut Català d'Oncologia. Barcelona, España E-mail: crodriguez@iconcologia.net

6 Claudia Villanueva. Institut d'Investigació Biomèdica de Bellvitge (IDIBELL), Barcelona, España. E-mail: cvillanueva@idibell.cat

7 Enric C. Sumalla. Institut d'Investigació Biomèdica de Bellvitge (IDIBELL), Barcelona, España E-mail: enricsumalla@gmail.com

8 Miquel Alabèrnia-Segura. Dpto. Psicología Clínica y Psicobiología, Universitat de Barcelona, Barcelona, España. E-mail: miquelalabernia@ub.edu

9 Claudia Prats. Institut d'Investigació Biomèdica de Bellvitge (IDIBELL). Dpto. de Biología Evolutiva, Ecología i Ciencias Ambientales, Universitat de Barcelona. Institut de Biomedicina de la Universitat de Barcelona (IBUB), Barcelona. Instituto de Salud Carlos III, Centro de Investigación Biomédica en Red de Salud Mental (CIBERSAM), Madrid. España

E-mail: cprats@idibell.cat

10 María José Corral. Dpto. Psicología Clínica y Psicobiología, Universitat de Barcelona. Institut de Neurociències, Universitat de Barcelona. Institut de Recerca Sant Joan de Déu, Esplugues de Llobregat, España.

E-mail:mjcorral@ub.edu

11 David Gallardo-Pujol. Dpto. Psicología Clínica y Psicobiología, Universitat de Barcelona. Institut de Neurociències, Universitat de Barcelona, Barcelona, España

E-mail: david.gallardo@ub.edu

12 Guillem Feixas. Dpto. Psicología Clínica y Psicobiología, Universitat de Barcelona. Institut de Neurociències, Universitat de Barcelona, Barcelona, España

E-mail: gfeixas@ub.edu

13 Candela Calle. Institut Català d'Oncologia, Barcelona, España.

E-mail: ccalle@iconcologia.net

14 Jordi Trelis. Institut Català d'Oncologia, Barcelona, España.

E-mail: jtrelis@iconcologia.net

15 Josep Maria Borràs.Depto.de Ciencias Clínicas, Universitat de Barcelona, Barcelona, España

E-mail: jmborras@iconcologia.net

* Dirección de correspondencia: Dr. Cristian Ochoa Arnedo, Programa E-Health ICOnnecta’t, Hospital Duran i Reynals, Institut Català d'Oncologia. Av. Gran Via de l'Hospitalet, 199-203, L'Hospitalet de Llobregat, 08908 Barcelona, Spain. Email: cochoa@iconcologia.net 
Resumen: Introducción: A pesar de que los tratamientos psico-oncológicos han demostrado su efectividad en disminuir el malestar emocional y mejorar la calidad de vida de las personas con cáncer, aún existen numerosas barreras que limitan su acceso. La transformación a online de esta atención se plantea como una solución para aumentar la cobertura del servicio y mejorar su coste-utilidad. Objetivo: Crear un ecosistema digital de salud para reducir el impacto del cáncer, aumentando el bienestar y la calidad de vida del ciudadano con cáncer. Método: Programa dirigido a pacientes diagnosticadas de cáncer de mama en fase de supervivencia aguda. Es un programa de atención escalonada dividido en 4 niveles de intervención jerarquizados por complejidad: Nivel 1, cribado y monitorización psicosocial; Nivel 2, Campus: psicoeducación y educación sanitaria; Nivel 3, soporte psicosocial comunitario; y Nivel 4, tratamiento psicoterapéutico grupal. Resultados: En 2019, 259 mujeres fueron incluidas en el programa (39,91\% de los nuevos casos de cáncer de mama en los centros participantes). Solo el 3,47\% $(\mathrm{n}=9)$ requirió atención clínica especializada (Nivel 4). Conclusión: El programa Iconnecta 't adopta un modelo integrado de atención psicosocial en cáncer que se adecúa a las necesidades específicas de los supervivientes. Da solución a algunas de las barreras de la atención sanitaria tradicional, democratizando el acceso a los servicios mediante el uso de tecnologías de uso común en la mayoría de ciudadanos. En un futuro próximo se prevé la implementación progresiva a otras neoplasias, junto con un ensayo clínico controlado y aleatorizado que evaluará su eficacia.

Palabras clave: e-Health; atención integral; prescripción digital; cáncer de mama; psicooncología; psicología de la salud.

\title{
[en] E-Health ICOnnecta't program: An ecosystem to promote wellbing in cancer towards ONCOMMUN European proposal
}

\begin{abstract}
Introduction: Although psycho-oncological treatments have already demonstrated their effectiveness in reducing emotional distress and improving quality of life in people with cancer, there are still numerous barriers limiting their access. The digital transformation of care is proposed as a solution to increase service coverage and improve its cost-utility. Aim: Create a digital health ecosystem to reduce the impact of cancer, increasing users' well-being and quality of life. Method: The program is addressed to patients diagnosed with breast cancer in the acute survival phase. It is a stepped-care intervention divided into 4 levels ordered by complexity: Level 1, psychosocial screening and monitoring; Level 2, Campus: psychoeducation and health education; Level 3, psychosocial community support; and Level 4, psychotherapeutic group treatment. Results: In 2019, 259 women were included in the program (39.91\% of new breast cancer cases in participating centers), and only $3.47 \%(n=9)$ required specialized clinical care (Level 4). Conclusions: The Iconnecta't program adopts an integrated model of psychosocial care in cancer that adapts to survivors' specific needs. It overcomes some of the barriers of traditional healthcare, democratizing service access through the use of common technologies among the vast majority of citizens. In the near future, progressive implementation to other cancer diagnoses is planned, together with a randomized controlled trial to evaluate its effectiveness.
\end{abstract}

Keywords: E-Health; comprehensive attention; Internet prescription,; breast cancer; psycho-oncology; health psychology

Sumario: 1. Introducción 2. Programa E-Health ICOnnecta't 2.1. Aspectos formales del programa. 2.2. Primer Nivel: Cribado y monitorización 2.3. Segundo Nivel: Campus: psicoeducación y educación sanitaria 2.4. Nivel 3: Soporte psicosocial comunitario 2.5. Nivel 4: Tratamiento psicoterapéutico grupal 3. Resultados 4. Conclusión 5. Agradecimientos 6. Financiación 7. Cumplimiento de los estándares éticos 8 . Referencias bibliográficas

Cómo citar: Ochoa-Arnedo C, Flix-Valle A, Medina JC, Escriche E, Rodríguez A, Villanueva C, Sumalla EC, Alabèrnia-Segura M, Prats C, Corral MJ, Gallardo-Pujol D, Feixas G, Calle C, Trelis J, Borràs JM. Programa E-Health ICOnnecta't: un ecosistema para fomentar el bienestar en cáncer a través de la propuesta europea ONCOMMUN. Psicooncología 2020;17:41-58. doi: 10.5209/psic.68240. 


\section{Introducción}

Se estima que en España hay más de 1.500 .000 supervivientes de cáncer, y que para el 2020 habrá un 50\% más ${ }^{(1)}$. Según el informe de 2020 de la Sociedad Española de Oncología Médica (SEOM) sobre "Las cifras del cáncer en España" se prevé que el cáncer de mama será, en datos hasta el 2018, el más prevalente a 5 años de todos los tumores, teniendo una tasa de supervivencia a los 5 años del $85,5 \%{ }^{(2)}$.

Sin embargo, estas altas tasas de supervivencia no implican necesariamente un mayor bienestar. Varios estudios muestran que los supervivientes de cáncer tienen más limitaciones funcionales, problemas psicosociales y laborales, y más miedo por su salud, en comparación con pacientes no oncológicos ${ }^{(3-6)}$. Específicamente relacionado con la respuesta psicológica a la enfermedad, ser diagnosticado de cáncer genera un malestar emocional significativo (principalmente síntomas de ansiedad y depresión) en un gran número de pacientes $(35 \% \text { a } 38 \%)^{(6-8)}$. Dicho malestar emocional es especialmente importante en las transiciones psicosociales de la enfermedad ${ }^{(9,10)}$, ya que son las fases más críticas en el proceso de adaptación psicosocial. Estas transiciones hacen referencia a la fase de supervivencia aguda (periodo entre el diagnóstico y los tratamientos oncológicos primarios) y de supervivencia extendida (periodo entre el fin de los tratamientos oncológicos y los primeros seguimientos de control) ${ }^{(10)}$. A pesar de la abundante evidencia que demuestra que el malestar emocional en estas fases se asocia con peor calidad de vida $(\mathrm{CV})$, menor adherencia a los tratamientos oncológicos, supervivencia global y adopción de estilos de vida saludables y autocuidado ${ }^{(11,12)}$, actualmente solo una pequeña proporción de pacientes son identificados por parte de los profesionales de oncología y remitidos a tratamiento psicosocial ${ }^{(13)}$.

El más reciente, amplio y completo meta-análisis y revisión sistemática realizado hasta la fecha, que abarca 22.238 pacientes, sobre los efectos de las intervenciones psicosociales sobre el malestar emocional y la CV en pacientes con cáncer, ha mostrado efectos positivos mantenidos en la reducción de malestar emocional, la ansiedad, la depresión y en la mejora de la $\mathrm{CV}$ asociada con la salud ${ }^{(9)}$. Aunque está bien establecido que la facilidad e idoneidad de acceso a un tratamiento psicooncológico puede mejorar la adaptación psicológica a la enfermedad, este acceso está lejos de ser universal, sobre todo en España ${ }^{(14)}$. Además de la escasez de psicooncólogos en el Sistema Nacional de Salud, se han detectado otros factores que limitan este acceso: pobre detección temprana; elevado tiempo de espera desde la demanda hasta la intervención; y restricciones por tiempo, trabajo, movilidad, o económicas de los pacientes para acudir presencialmente a los centros ${ }^{(15)}$.

Los países con mayores recursos económicos y una mejor atención integral en cáncer recomiendan el uso sistemático de un cribado de malestar emocional y psicosocial para identificar a pacientes con mayor riesgo físico, psicológico o social que pueda interferir en el acceso a servicios y cuidados claves, su adherencia terapéutica o el uso innecesario de los recursos sanitarios ${ }^{(16,17)}$. Disponer de un protocolo de cribado y monitorización psicosocial, que garantice que los pacientes en riesgo pueden beneficiarse a tiempo de un tratamiento psicosocial con evidencia empírica, es un estándar necesario para la acreditación de centros oncológicos americanos y europeos, sin embargo, su implementación es escasa, inconsistente y muy irregular ${ }^{(15,18)}$.

Una razón clave para la complicada implementación del cribado y monitorización psicosocial es que los proveedores habituales de cuidados y tratamientos oncológicos 
(enfermería y oncología médica) perciban estos instrumentos como clínicamente útiles y orientados a poder garantizar una atención psicosocial posterior ${ }^{(19)}$. Cribar sin ofrecer después un tratamiento psicosocial oportuno es una de las principales críticas a los sistemas de cribado psicosocial actuales ${ }^{(20)}$.

En este sentido, las actuales Tecnologías de la Información y Comunicación (TICs) están consiguiendo dar solución a buena parte de todos estos problemas de acceso e implementación de sistemas de cribado, monitorización y tratamiento psicosocial; pero siguen sin estar integradas en las estructuras de salud pública. Asimismo, no hemos encontrado ningún estudio en la literatura que integre estos sistemas, escalone las intervenciones por niveles de complejidad, realice todo el proceso "vía online" y calcule su eficacia y coste-efectividad. Teniendo en cuenta el aumento del diagnóstico de cáncer y sus elevadas tasas de supervivencia, la integración tecnológica de sistemas de cribado, monitorización y escalamiento de las intervenciones psicosociales se plantea como una solución para aumentar la cobertura del servicio y mejorar su coste-utilidad ${ }^{(21)}$. Revisiones muy recientes sobre el coste-efectividad y el coste-utilidad del tratamiento psicosocial consideran que este es probablemente coste-efectivo y aceptable por los pagadores de los servicios, pero plantea dudas sobre su implementación a gran escala en cuanto a accesibilidad, aceptabilidad de los usuarios y sincronía de la demanda-intervención ${ }^{(22)}$.

Paralelamente, si atendemos al uso que hacen de internet los pacientes oncológicos, los estudios indican que un 70-97\% de ellos buscan información médica online $\left.{ }^{(23,24}\right)$. Este uso de internet se incrementa significativamente después del diagnóstico y se mantiene en la fase de supervivencia extendida, lo que indicaría necesidades de información no cubiertas ${ }^{(25)}$. Y, a pesar de que los pacientes prefieren consultar webs estáticas de información médica, pasan mucho más tiempo en comunidades dinámicas, como redes sociales, lo que indicaría la necesidad de estar socialmente conectados ${ }^{(25,26)}$. Finalmente, cabe señalar que los pacientes no comparten la información que consultan por internet con su equipo sanitario por miedo a dañar la relación terapéutica de confianza. Al mismo tiempo, los sanitarios minimizan el potencial de internet para crear conocimiento de calidad y maximizan el impacto psicológico negativo que tiene esa información sobre los pacientes ${ }^{(25,27)}$.

En este contexto, nuestro equipo del Institut Català d'Oncologia (ICO) desarrolló, en el 2019, el programa e-Health ICOnnecta 't; el primer ecosistema digital de salud para construir espacios de comunicación online y fomentar experiencias saludables en cáncer, integrando un programa psicosocial con cuatro niveles de intervención. Estos niveles abarcan desde la prevención y promoción educativa de la salud (primer y segundo nivel), las intervenciones más comunitarias (tercer nivel), hasta las más personalizadas, profesionalizadas e intensivas (cuarto nivel).

\section{Programa E-Health ICOnnecta't}

Antes de detallar el programa, primero debemos explicar qué entendemos por e-Health. Ésta se define como el conjunto de Tecnologías de la Información y la Comunicación (TICs) que se emplean en el entorno sanitario para la prevención, diagnóstico, tratamiento, seguimiento y gestión de la salud, con el objetivo de facilitar el acceso, reducir costes, mejorar la eficacia o la calidad de los servicios sanitarios $^{(28)}$. Aplicaciones móviles, teleasistencia, dispositivo "wereables" que se 
integran en la ropa, o videojuegos de salud, son algunos ejemplos de productos y servicios e-Health.

El programa ICOnnecta 't es la materialización en el Insituto Catalán de Oncologia del proyecto europeo "ONCOMMUN: Online Cancer Support Communities" (Comunidades de Soporte Online en Cáncer) (https://oncommun.eu/?lang=es) apoyado por el European Institute of Innovation and Technology (EIT Health). En él participan más de 10 instituciones de 4 países (Alemania, Portugal, Polonia y España). Entre estas otras instituciones encontramos: el Hospital de la Santa Creu i Sant Pau (Barcelona); el Hospital General Universitario Gregorio Marañón (Madrid); el Instituto Portuguese de Oncologia Coimbra en colaboración con el Instituto Pedro Nunes (Portugal); y el Copernicus Memorial Hospital en colaboración con el Nofer Institute of Occupational Medicine (Polonia).

La misión de ICOnnecta't es crear un ecosistema digital e-Health que conecte y facilite la comunicación entre pacientes y profesionales sanitarios con el objetivo de reducir el impacto del cáncer, aumentando el bienestar y la calidad de vida durante el seguimiento y atención continuada del ciudadano con cáncer. Para alcanzar esta misión, el ecosistema e-Health dispone de un programa de atención psicosocial y educativo basados en un sistema de cribado, monitorización y atención online escalonado, que permite una detección e intervención psicosocial temprana. En particular, los objetivos específicos de esta atención psicosocial online son los siguientes: a) facilitar el acceso seguro a información de calidad sobre cáncer, cocreada por un equipo multidisciplinar y los usuarios; b) monitorizar de forma sostenida variables psicosociales para ofrecer un soporte psicosocial y apoyo emocional ajustado a necesidades específicas detectadas; c) el acceso a un tratamiento psicooncológico online basado en la evidencia, y d) calcular el coste-utilidad y costeefectividad de las intervenciones psicosociales online. Numerosos estudios muestran como este tipo de intervención facilita el proceso de adaptación a la enfermedad y aumenta la calidad de vida de las personas con un diagnóstico oncológico ${ }^{(18,29-31)}$, además de mejorar el control sintomático ${ }^{(32,33)}$, la comunicación con los profesionales sanitarios y la satisfacción de los propios pacientes ${ }^{(33,34)}$.

\subsection{Aspectos formales del programa}

\section{Participantes}

El programa va dirigido a pacientes en fase de supervivencia aguda diagnosticadas de cáncer de mama. La inclusión de pacientes se inició en marzo de 2019. Toda mujer atendida en las Unidades Funcionales de Mama de los tres centros ICO (Hospitalet, Badalona y Girona) que estuviera en tratamiento oncológico primario se le invitaba a participar. En marzo de 2020 se iniciará la inclusión de pacientes con enfermedades onco-hematológicas (leucemia mieloide aguda, leucemia mieloide crónica y mieloma múltiple). En un futuro se prevé la implementación progresiva en cáncer de pulmón avanzado y otras neoplasias. El presente trabajo se centra en detallar las características del programa implementado en el Instituto Catalán de Oncología (ver tabla 1) ya que es la institución desarrolladora del programa y la que lo implementa con el mayor número de pacientes. 
Tabla 1. Características del programa e-Health ICOnnecta't

\begin{tabular}{lll}
\hline Centros & - & Institut Català d'Oncologia L'Hospitalet (Hospital Duran i Reynals) \\
participantes & - & Institut Català d'Oncologia Badalona (Hospital Germans Trias i Pujol) \\
& - & Institut Català d'Oncologia Girona (Hospital Josep Trueta de Girona) \\
\hline Criterios de & - & Pacientes adultas ( $\geq 18$ años) \\
inclusión & - & Diagnóstico de cáncer de mama en las 6 semanas previas a la inclusión \\
& - & Tener teléfono móvil o tableta con acceso a internet y conocimiento de \\
& & estas herramientas a nivel usuario \\
\hline Criterios de & Padecer alguno de los siguientes: \\
exclusión & - & Episodio depresivo mayor \\
& - & Ideación autolítica significativa \\
& - & Síntomas psicóticos activos \\
\hline Criterios para & - & Trastorno por abuso de substancias \\
abandonar el & - & Problemas tecnológicos continuados \\
programa & - & No adherencia al programa \\
& - & Solicitud de apoyo psicooncológico presencial justificada. \\
& - & Presencia de malestar emocional elevado después de seguir los cuatro \\
& & niveles de intervención del programa \\
\hline Equipo & \multicolumn{2}{l}{ Profesionales sanitarios que participan directamente en el programa: } \\
interdisciplinar & Psicología \\
& - & Enfermería \\
& - & Psiquiatría \\
& - & Psicopedagogía \\
& - & Trabajo social \\
& - & Oncología \\
\hline Intervención & - & Farmacia \\
\hline Intervención psicosocial escalonada según necesidades detectadas: & - & Nivel 1: Cribado y monitorización psicosocial \\
& - & Nivel 2: Campus: psicoeducación y educación sanitaria \\
& - & Nivel 3: Soporte psicosocial comunitario \\
& - & Nivel 4: Tratamiento psicoterapéutico grupal \\
\hline & &
\end{tabular}

Sistema escalonado de atención psicosocial y educativa

El programa ICOnnecta't se crea en el ámbito psicosocial del cáncer como un ecosistema digital escalonado de educación y atención sanitaria basado en 4 niveles de intervención jerarquizados por intensidad y complejidad. Este sistema representado en una pirámide (ver figura 1), refleja un modelo integral de atención y gestión sanitaria. Cada nivel de atención se corresponde con una solución y funcionalidad tecnológica diferente que utiliza sistemas de comunicación e intervención bien establecidos para un usuario de internet básico. La tabla 2 muestra los objetivos y recursos tecnológicos del programa según los niveles de intervención.

El primer nivel de atención es una herramienta de cribado y monitorización online (Nivel 1), seguida de un campus educativo (Nivel 2), una comunidad de soporte psicosocial (Nivel 3) y tratamiento psicoterapéutico grupal a través de multivideoconferencia (Nivel 4). Los primeros tres niveles han sido las intervenciones más frecuentemente investigadas en la literatura. Consisten generalmente en el uso de foros asincrónicos para el intercambio de información, opiniones y apoyo; 
Figura 1. Intervención escalonada del programa e-Health ICOnnecta’t

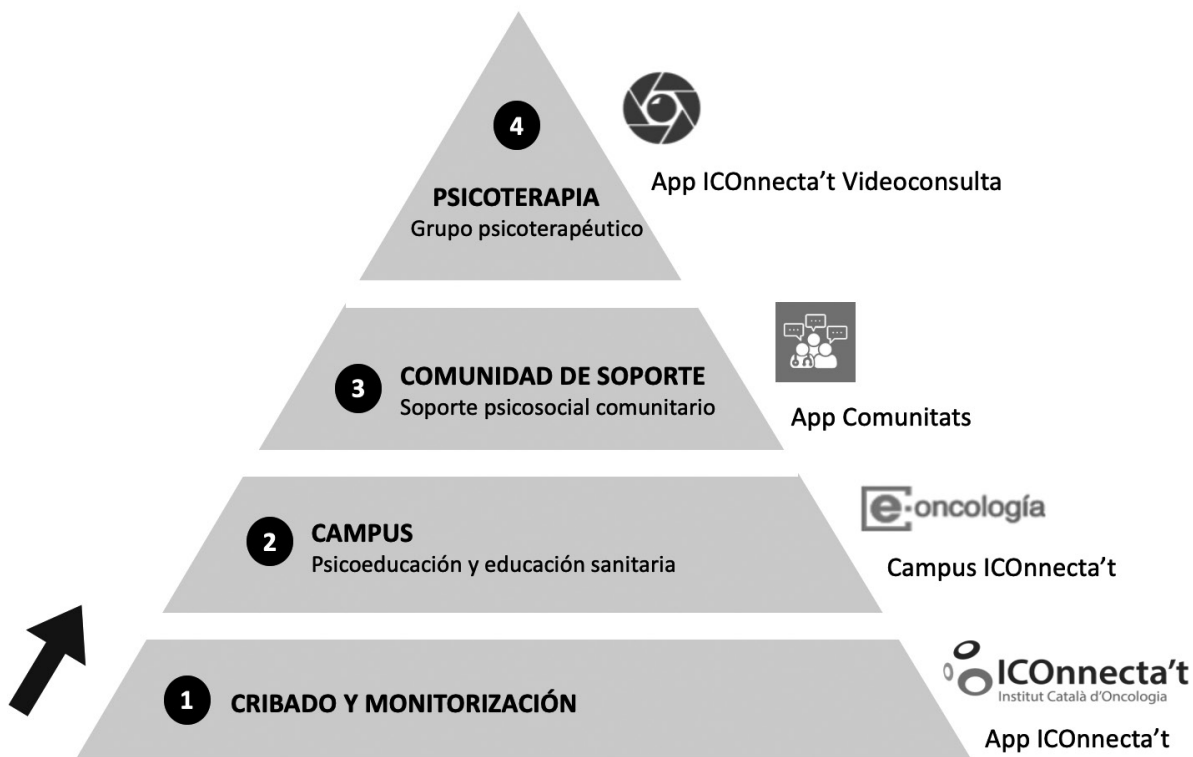

Tabla 2. Objetivos y recursos tecnológicos del programa e-Health ICOnnecta 't según niveles de intervención

\begin{tabular}{|c|c|c|c|}
\hline $\begin{array}{c}\text { Nivel de } \\
\text { intervención }\end{array}$ & Objetivo general & Objetivos específicos & $\begin{array}{l}\text { Recurso } \\
\text { tecnológico }\end{array}$ \\
\hline $\begin{array}{l}\text { Nivel } 1 \\
\text { Cribado y } \\
\text { monitorización } \\
\text { psicosocial }\end{array}$ & $\begin{array}{l}\text { Detección, } \\
\text { monitorización y } \\
\text { atención continuada } \\
\text { de necesidades } \\
\text { psicosociales y de } \\
\text { salud. }\end{array}$ & $\begin{array}{l}\text { - Detección y seguimiento } \\
\text { proactivo de las medidas } \\
\text { reportadas por los pacientes } \\
\text { (Patient Reported Outocomes } \\
\text { measures) que inciden en el } \\
\text { proceso oncológico y son } \\
\text { susceptibles de control y manejo } \\
\text { remoto. } \\
\text { - Plan educativo o terapéutico } \\
\text { individualizado y pro-activo, } \\
\text { basado en la monitorización y } \\
\text { detección. }\end{array}$ & $\begin{array}{l}\text { App } \\
\text { ICOnnecta't: } \\
\text { plataforma para } \\
\text { monitorizar los } \\
\text { Patient Reported } \\
\text { Outocomes } \\
\text { measures }\end{array}$ \\
\hline $\begin{array}{l}\text { Nivel } 2 \\
\text { Campus: } \\
\text { psicoeducación y } \\
\text { educación sanitaria }\end{array}$ & $\begin{array}{l}\text { Educación sanitaria } \\
\text { digital mediante } \\
\text { recursos co-creados } \\
\text { por un equipo } \\
\text { multidisciplinar. }\end{array}$ & $\begin{array}{l}\text { - Educación sanitaria } \\
\text { personalizada. } \\
\text { - Prescripción digital de recursos } \\
\text { online validados. } \\
\text { - Seguimiento por parte del } \\
\text { profesional del plan educativo o } \\
\text { terapéutico individualizado. }\end{array}$ & $\begin{array}{l}\text { Campus } \\
\text { ICOnnecta't } \\
\text { (E-Oncología): } \\
\text { moodle } \\
\text { educativo }\end{array}$ \\
\hline
\end{tabular}




\begin{tabular}{|c|c|c|c|}
\hline $\begin{array}{c}\text { Nivel de } \\
\text { intervención }\end{array}$ & Objetivo general & Objetivos específicos & $\begin{array}{l}\text { Recurso } \\
\text { tecnológico }\end{array}$ \\
\hline $\begin{array}{l}\text { Nivel } 3 \\
\text { Soporte psicosocial } \\
\text { comunitario }\end{array}$ & $\begin{array}{l}\text { Soporte psicosocial } \\
\text { entre pacientes y entre } \\
\text { paciente-profesional } \\
\text { dentro de una } \\
\text { comunidad online, } \\
\text { en forma de red } \\
\text { social privada, para } \\
\text { establecer una alianza } \\
\text { social y terapéutica } \\
\text { digital. }\end{array}$ & $\begin{array}{l}\text { - Apoyo emocional y social } \\
\text { mutuo. } \\
\text { - Compartir información y } \\
\text { contrastarla con profesionales. } \\
\text { - Prescripción digital de recursos } \\
\text { online validados. }\end{array}$ & $\begin{array}{l}\text { Web/App } \\
\text { Comunitats: red } \\
\text { social privada }\end{array}$ \\
\hline $\begin{array}{l}\text { Nivel } 4 \\
\text { Tratamiento } \\
\text { psicoterapéutico } \\
\text { grupal }\end{array}$ & $\begin{array}{l}\text { Tratamiento } \\
\text { psicoterapéutico cara } \\
\text { a cara a mediante } \\
\text { teleasistencia (multi- } \\
\text { videoconferencia). }\end{array}$ & $\begin{array}{l}\text { - Telemedicina cara a cara. } \\
\text { - Intervenciones personalizadas } \\
\text { que mimetizan las consultas } \\
\text { presenciales que no requieren } \\
\text { exploración física (salvo la } \\
\text { visual). }\end{array}$ & $\begin{array}{l}\text { Web/App } \\
\text { ICOnnecta't } \\
\text { Videoconsulta: } \\
\text { plataforma de } \\
\text { videoconsultas }\end{array}$ \\
\hline
\end{tabular}

o la creación de sitios web diseñados para apoyar a los pacientes con cáncer en el autocontrol de su enfermedad, sin supervisión psico-oncológica ${ }^{(35)}$. En nuestro sistema, estos tres niveles se han incluido como estrategias de prevención y promoción de la salud dada la necesidad de información relacionada con la salud y el apoyo emocional entre los sobrevivientes reportada por estudios previos ${ }^{(24,25)}$. En cuanto al tratamiento psicoterapéutico del último nivel, en los últimos años, algunos ensayos clínicos controlados y aleatorizados han evaluado esta nueva modalidad de terapia, y sus primeros resultados comienzan a revelar una eficacia equivalente al formato presencial en el tratamiento del malestar emocional y la psicopatología, así como en el aumento de la calidad de vida ${ }^{(36,37)}$.

\subsection{Primer Nivel: Cribado y monitorización}

\section{Cribado, monitorización psicosocial y sistema de escalado por niveles}

Mediante la aplicación móvil central del programa (App ICOnnecta't), se realiza el cribado y monitorización de diversas variables psicosociales (ver tabla 3). Los pacientes reciben una alerta en su dispositivo (agenda del paciente) para que registren e informen de dichas variables, que han sido prescritas y programadas previamente por el profesional sanitario. Éstos tienen acceso a toda la información reportada en tiempo real mediante una plataforma web de monitorización. De esta manera puede seguirse fácilmente la evolución del malestar emocional, el estrés postraumático u otros indicadores de salud como la CV o el bienestar. 
Tabla 3. Cuestionarios para el cribado y monitorización psicosocial (Nivel 1)

\begin{tabular}{lll}
\hline \multicolumn{1}{c}{ Variable psicosocial } & \multicolumn{1}{c}{ Cuestionario } & Frecuencia \\
\hline Estado emocional & Termómetro emocional (TE) 0-10 & Semanal \\
Malestar emocional & Hospital Anxiety and Depression Scale (HADS) & $\begin{array}{l}\text { Tras TE }>5 \text { y } \\
\text { cada } 3 \text { meses }\end{array}$ \\
Calidad de vida & European Quality of Life-5Dimensions (EQ-5D) & Cada 3 meses \\
Estrés postraumático & Post-traumatic stress disorder checklist (PCL-5) & Cada 3 meses \\
Crecimiento postraumático & Post-traumatic growth inventory (PTGI) & Cada 3 meses \\
Apoyo social & The MOS Social Support Survey (MOS) & Cada 6 meses \\
Bienestar & Pemberton Happiness Index (PHI) & Cada 6 meses \\
Satisfacción con la plataforma & Escala visual analógica 0-10 & Cada 6 meses \\
Usabilidad de la aplicación & System Usability Scale (SUS) & Cada 6 meses \\
Insomnio & Insomnia Severity Index (ISI) & Si procede \\
\hline
\end{tabular}

Las puntuaciones obtenidas durante la monitorización es lo que permite cribar e implementar la intervención escalonada. Cuando el Termómetro Emocional (TE), pautado semanalmente, puntúa entre 5-10 (siendo 0 la no presencia de malestar, y 10 el máximo malestar posible) durante dos semanas consecutivas, entonces se administra el Hospital Anxiety and Depression Scale (HADS). Si se obtiene una puntuación superior a 10 (puntuaciones entre 11-16 denotan malestar emocional a considerar, y puntuaciones entre 17-42 implican malestar emocional clínicamente significativo), entonces la paciente será escalada al nivel 2 de intervención. Para ello, un psicólogo general sanitario realiza una videoconsulta (App ICOnnecta't Videoconsulta) con la paciente para explorar el malestar emocional reportado y los problemas psicosociales asociados. De esta manera podrán prescribirse aquellos recursos psicoeducativos o de educación sanitaria del nivel 2 que estén directamente relacionados con dicho malestar. El mismo procedimiento se sigue para pasar al nivel 3 y posteriormente al 4 , permaneciendo y monitorizando a la paciente en cada uno de los niveles durante al menos dos semanas. Resaltar que todos los movimientos hacia los niveles de intervención son precedidos por una videoconsulta con uno de los profesionales ICOnnecta't de referencia de la paciente para acompañarla y guiarla durante todo el proceso.

\section{Cribado y monitorización de enfermería para educación sanitaria}

La misma aplicación móvil central permite a las pacientes informar de los signos y síntomas relacionados con los efectos secundarios y complicaciones de los tratamientos oncológicos. Al registrar un síntoma, la aplicación hace una serie de preguntas clave en forma de árbol de decisión siguiendo el protocolo $\mathrm{CTCAE}^{(38)}$ para establecer el nivel de gravedad y toxicidad del síntoma experimentado. Cuando el paciente acaba de responder las preguntas, inmediatamente, la aplicación muestra consejos de educación sanitaria validados para ayudar a los pacientes a gestionar dicho síntoma 
de forma básica y/o remitirle a algún recurso de urgencia si fuera necesario. Los consejos sanitarios están adaptados a la gravedad reportada, por lo que solo reciben la información adecuada a sus problemas específicos de salud evitando sobreinformación o consejos no adaptados a sus necesidades. Mediante la plataforma web del profesional, enfermería puede monitorizar los niveles de toxicidad en el momento que aparecen y ponerse en contacto con la paciente mediante videoconferencia o llamada telefónica si se detectan niveles significativos de esta toxicidad.

\subsection{Segundo Nivel: Campus: psicoeducación y educación sanitaria}

En los dos siguientes niveles de intervención, se ofrecen recursos educativos para aumentar el conocimiento sobre la enfermedad y potenciar estrategias de afrontamiento que faciliten la construcción de experiencias saludables en cáncer.

En el Nivel 2 encontramos el Campus ICOnnecta't, el cual se accede mediante la App central. Se trata de una plataforma educativa Moodle donde las pacientes pueden consultar vídeos y recursos online con información fiable y rigurosa de las diferentes etapas del proceso oncológico. Los recursos disponibles son co-creados por un equipo multidisciplinar del ICO y coordinado por una psicopedagoga y ex-paciente. Los profesionales del ICO los seleccionan y desarrollan atendiendo a las demandas de información que los propios pacientes informan durante sus tratamientos y/o mediante la monitorización del Nivel 1. Hasta la fecha, el Campus cuenta con 23 vídeos, 20 artículos web, 12 documentos descargables y 6 infografías. Todo ello dividido en 6 bloques temáticos: mis emociones, tratamientos médicos, mis relaciones personales, mi cuerpo, vida saludable, y mí día a día. Todo el material de Campus está disponible también en la página web de acceso libre del equipo ICOnnecta 't ${ }^{(39)}$.

Existen dos metodologías educativas no excluyentes en este nivel de intervención:

a) Metodología espontánea: las pacientes acceden libremente al contenido del Campus desde el momento en que son incluidas en el programa, pudiendo consultar todo aquel material que sea de su interés.

b) Metodología guiada: los profesionales que monitorizan a las pacientes pueden ir prescribiendo aquellos temas y recursos específicos congruentes con las necesidades psicosociales y/o de educación sanitaria detectadas en el primer nivel del programa mediante o la videoconsulta. Para conocer si la paciente ha seguido las pautas educativas, el profesional puede verificar qué material ha consultado mediante la plataforma web de seguimiento. Para evaluar la adquisición del conocimiento, el Campus permite realizar una evaluación global y cualitativa mediante 38 pequeños cuestionarios dispuestos en cada uno de los recursos. De esta forma se garantiza el cumplimiento del itinerario formativo individual y personalizado propuesto.

En un futuro se pretende evaluar qué metodología educativa ofrecer mayor valor y beneficios psicoeducativos a las pacientes.

Después de la prescripción de recursos del Campus, el psicólogo del programa sigue monitorizando a la paciente durante al menos dos semanas. Si el malestar emocional o las necesidades psicosociales no disminuyen, se vuelve a contactar con ella mediante videoconferencia para dirigirla al siguiente escalón del programa, la intervención comunitaria. 


\subsection{Nivel 3: Soporte psicosocial comunitario}

El tercer nivel está formado por una comunidad de soporte psicosocial para cáncer de mama (App Comunitats) que pretende generar una alianza terapéutica digital entre pacientes y entre paciente-profesional. Esta comunidad ofrece un entorno seguro donde compartir experiencias y hablar sobre temas relacionados con la enfermedad de forma anónima. Funciona como una red social privada con diferentes foros de debate (bloques temáticos) donde pueden encontrarse también los recursos educativos del Campus ICOnnecta't. Los bloques temáticos tienen una estructura similar a los del Campus. Distintos profesionales del programa supervisan y hacen un seguimiento de las contribuciones, dando respuesta profesional si se precisa (los profesionales sanitarios aparecen identificados con su nombre, fotografía y especialidad). Las usuarias para garantizar su confidencialidad aparecen con un pseudónimo o "nickname". El sistema tecnológico tiene un funcionamiento muy similar a otras tecnologías bien establecidas en la sociedad (ej. App Whatsapp).

La comunidad online se enmarca dentro de la línea estratégica para el fomento de la participación ciudadana en salud de la Generalitat de Catalunya. Al ser una aplicación avalada por el Departament de Salut, en colaboración con el ICO, todos los pacientes con cáncer de Cataluña, de los que se disponga una comunidad, pueden tener acceso a esta aplicación en cualquier momento del proceso de su enfermedad, también en la fase de supervivencia extendida. No hace falta ser usuario de todo el programa ICOnnecta't para ser usuario de la misma.

De igual manera que en el nivel anterior, después de prescribir a la paciente el Nivel 3, se la sigue monitorizando durante al menos dos semanas. Si el malestar emocional o las necesidades psicosociales no disminuyen, se contactar de nuevo con la paciente vía videoconferencia para dirigirla al último Nivel del programa, el más personalizado, profesionalizado e intensivo.

\subsection{Nivel 4: Tratamiento psicoterapéutico grupal}

El último Nivel lo configura el tratamiento psicoterapéutico grupal por multivideoconferencia (App ICOnnecta't Videoconsulta) aplicado por un psicólogo clínico especialista, experto en psico-oncología, y se dirige a aquellos pacientes con malestar emocional clínicamente significativo y dificultades moderadas o graves de adaptación (ver tabla 4). Dicho tratamiento se basa en el programa de Psicoterapia Positiva Grupal en cáncer diseñado para supervivientes al finalizar los tratamientos oncológicos primarios. El manual de este programa de tratamiento grupal ha sido publicado tanto en español ${ }^{(40)}$, como en inglés ${ }^{(41)}$. Se sigue un modelo psicoterapéutico que integra elementos cognitivoconductuales y humanístico-experiencial desde una perspectiva de construcción de narrativas terapéuticas ${ }^{(42)}$. Su objetivo es facilitar el crecimiento postraumático a través de los métodos psicoterapéuticos asociados al desarrollo de cambios positivos como son emociones positivas, fortalezas y sentido y significado vital ${ }^{(40)}$.

En su formato presencial, este tratamiento grupal ha demostrado ser más efectivo en la reducción del malestar emocional y el estrés postraumático y en la mejora de la calidad de vida que una terapia grupal cognitivo conductual de manejo del estrés ${ }^{(43)}$ o un grupo de lista de espera ${ }^{(44)}$. Por último, un reciente ensayo clínico controlado y aleatorizado ha demostrado que el formato online presenta el mismo nivel de eficacia que el formato presencial ${ }^{(37)}$. 
Tabla 4. Características del tratamiento psicoterapéutico grupal por multi-videoconferencia (Nivel 4). Adaptado del programa de Psicoterapia Positiva Grupal en cáncer ${ }^{(40)}$

- Intervención clínica orientada a pacientes en tratamiento oncológico activo

- Teleasistencia: Multi-videoconferencia (App ICOnnecta’t Videoconsulta)

- Grupo dirigido por un psicólogo clínico (más un observador)

- $\quad 8$ sesiones con dos seguimientos a los 3 y 12 meses, 90-120 min. de duración, frecuencia semanal

- Grupo cerrado

- 5-7 pacientes

- Requisitos tecnológicos mínimos: ordenador o teléfono móvil con acceso a internet, cámara web y conocimiento básico del uso de internet

\section{Resultados}

\section{Análisis descriptivo y preliminar de implementación del programa}

Durante el 2019, el programa ICOnnecta't atendió un total de 566 usuarias. De ellas, 307 fueron usuarias de toda Catalunya incluidas únicamente en la comunidad de soporte psicosocial (Nivel 3), mientras que 259 fueron mujeres diagnosticadas de cáncer de mama en el ICO e incluidas en el programa de atención escalonada completo. Si atendemos a esta última cifra, y teniendo en cuenta que en 2019 se diagnosticaron 649 nuevos casos de cáncer de mama en los hospitales participantes, se puede establecer que el programa ha dado cobertura al 39,91\% de los nuevos casos. También cabe resaltar que el $70,27 \%(n=182)$ de las pacientes eran pacientes activas con un uso semanal de la App central ICOnnecta't, lo que nos permitió monitorizar y detectar fácilmente sus necesidades psicosociales para ofrecer una atención escalonada y personalizada. Resultados que concuerdan con la literatura actual, donde la tasa media de abandono de los programas de tratamiento psicológico online se sitúa alrededor del 31\% (45). Respecto a la atención psicosocial ofrecida, en tan solo 3 días de promedio (incluidos los fines de semana) se dio respuesta a las necesidades psicosociales detectadas. Por último, es importante señalar que el ecosistema digital ha demostrado, hasta la fecha, una alta capacidad para resolver posibles casos clínicos de forma preventiva, ya que solo el 3,47\% $(n=9)$ de las pacientes incluidas requirió el nivel más alto de atención clínica especializada (Nivel 4).

\section{Impacto esperado, en proceso de evaluación}

Los datos ofrecidos hay que interpretarlos con cautela. Dado el escaso tiempo que el programa lleva implementándose, todavía no se disponen de suficientes datos empíricos que evalúen su eficacia ni su impacto sobre el bienestar, la calidad de vida $\mathrm{u}$ otros indicadores importantes en salud. Los resultados esperados pueden dividirse en tres grandes áreas de impacto:

- Primer impacto potencial: Mejorar el acceso a educación sanitaria de calidad y a atención psicosocial en cáncer. 
1. Reducir el tiempo de detección de necesidades de educación sanitaria y psicosociales en un $30 \%$.

2. Optimizar el tiempo de espera para recibir atención sanitaria para tales necesidades en un $25 \%$.

- Segundo impacto potencial: Implementar la primera evaluación de efectividad de un ecosistema digital de salud comparado con la atención psicosocial presencial habitual a nivel mundial.

3. Disminuir el malestar emocional y el estrés postraumático, y aumentar el crecimiento postraumático y la calidad de vida, en más del $50 \%$ de los supervivientes.

4. Resolver el $70 \%$ de los casos con riesgo psicosocial mediante intervenciones preventivas.

5. Mejorar los conocimientos de salud y en autocuidado en un $60 \%$ de los supervivientes.

6. Altos niveles de aceptabilidad de la plataforma, calificada como utilizable por más del $60 \%$ de los usuarios. Además, se espera que más del $80 \%$ estén satisfechos con ella.

- Tercer impacto potencial: Proporcionar los primeros resultados del impacto económico y ecológico de un ecosistema de salud digital.

7. Reducir los gatos asociados a la atención presencial habitual en un $30 \%$, logrando una mejor relación coste-utilidad por "años de vida ajustados por calidad" (AVAC) mediante la plataforma e-Health en al menos un $15 \%$.

8. Fomentar la adherencia a los fármacos oncológicos en el $70 \%$ de los supervivientes, y reducir en un $20 \%$ la medicación psicofarmacológica prescrita.

9. Reducir los días de baja laboral por enfermedad hasta en un $30 \%$ una vez finalizados los tratamientos oncológicos primarios.

\section{Ensayo clínico ICOnnecta't}

En los próximos meses se iniciará un ensayo clínico multicéntrico controlado y aleatorizado. Se comparará el ecosistema digital ICOnnecta't, que remite a una atención psicosocial y de control de síntomas integrada y escalonada íntegramente online, con un tratamiento habitual equivalente durante la fase de supervivencia aguda. Se evaluarán los resultados de ambas intervenciones en la detección, tiempo de espera y la proporción de casos resueltos en cada nivel. Así mismo, se valorará la eficacia diferencial en mejorar los niveles de malestar emocional, $\mathrm{CV}$, estrés postraumático y crecimiento postraumático. Por último, se realizará un análisis económico comparado de otros parámetros psicosociales y de salud relevantes como el tiempo de reincorporación laboral, la adherencia farmacológica y el uso de psicofármacos.

\section{Conclusión}

El programa ICOnnecta't aborda uno de los principales desafíos que plantea la sociedad digital actual en enfermedades graves como el cáncer: ¿cómo construir una alianza terapéutica y social entre los ciudadanos y sus proveedores de salud para garantizar una educación, manejo, empoderamiento y atención sanitaria 
continuada y sostenible? Desde el proyecto europeo ONCOMMUN proponemos un ecosistema digital basado en una atención psicosocial y sanitaria escalonada por niveles de complejidad que abarca desde la prevención y promoción de la salud, intervenciones comunitarias, hasta intervenciones psicoterapéuticas intensivas. Sin embargo, aún no existe evidencia empírica sobre la efectividad y coste-utilidad de este tipo de atención digital comparada con la atención presencial habitual. El primer año de implementación del programa servirá de experiencia para llevar a cabo el estudio clínico que lo evalúe; así como desarrollar una versión para enfermedades onco-hematológicas, cáncer de pulmón y otras neoplasias. En conclusión, este proyecto pretende avanzar hacia un modelo integrado de atención psicosocial en cáncer que se adapte a las necesidades específicas de los supervivientes. Tal reto tiene el potencial para convertirse en un punto de inflexión en la atención centrada en el paciente.

\section{Agradecimientos}

Agradecemos a todos los pacientes y sus familiares por su participación en este estudio, y al Programa CERCA por su apoyo institucional.

\section{Financiación}

Este estudio ha sido financiado por el Instituto Europeo de Innovación y Tecnología (EIT Health) (19046- ONCOMMUNITIES: Online Cancer Support Communities). EIT Health es la mayor red europea de innovación en salud. EIT Health cuenta con el apoyo del EIT, un organismo de la Unión Europea. Este trabajo también ha sido parcialmente apoyado por el Instituto de Salud Carlos III bajo la subvención FIS PI15/01278, cofinanciada por el Fondo Europeo de Desarrollo Regional (FEDER) "a way to build Europe". Grupo de investigación consolidado: Recerca en serveis sanitaris en cáncer, 2017SGR00735.

\section{Cumplimiento de los estándares éticos}

Conflicto de intereses

Los autores declaran que no hubo conflicto de intereses.

Aprobación ética

Todos los procedimientos realizados cumplieron con los estándares éticos de los comités institucionales relevantes sobre experimentación humana, y con la Declaración de Helsinki revisada en 2008.

\section{Consentimiento informado}

Todos los usuarios atendidos dieron su consentimiento informado previo a su inclusión en el programa. 


\section{Referencias bibliográficas}

1. Sociedad Española de Oncología Médica. Monográfico SEOM de Largos Supervivientes en Cáncer. Madrid: Sociedad Española de Oncología Médica (SEOM); 2012.

2. Sociedad Española de Oncología Médica. Las cifras del cáncer en España 2020 [Internet]. Sociedad Española de Oncología Médica (SEOM); 2020. [Acceso 1 de marzo de 2020]. Disponible en: https://seom.org/seomcms/images/stories/recursos/Cifras del_cancer_2020.pdf

3. Ahn SH, Park BW, Noh DY, Nam SJ, Lee ES, Lee MK, et al. Health-related quality of life in disease-free survivors of breast cancer with the general population. Ann Oncol 2007:173-82. doi: 10.1093/annonc/mdl333

4. Hoffman KE, McCarthy EP, Recklitis CJ, Ng AK. Psychological distress in long-term survivors of adult-onset cancer. Arch Intern Med 2009;169:1274-81. doi: 10.1001/ archinternmed.2009.179

5. Jarrett N, Scott I, Addington-hall J, Amir Z, Brearley S, Hodges L, et al. Informing future research priorities into the psychological and social problems faced by cancer survivors: A rapid review and synthesis of the literature. Eur J Oncol Nurs 2013;17:510 20. doi: 10.1016/j.ejon.2013.03.003

6. Knobf MT. Clinical Update: Psychosocial Responses in Breast Cancer Survivors. Semin Oncol Nurs 2011;27:e1-w14. doi: 10.1016/j.soncn.2011.05.001

7. Schumacher JR, Palta M, Loconte NK, Heidrich SM, Smith MA. Characterizing the psychological distress response before and after a cancer diagnosis. J Behav Med 2013;36:591-600. doi: 10.1007/s10865-012-9453-x

8. Zabora J, Brintzenhofeszoc K, Curbow B, Hooker C, Piantadosi S. The prevalence of psychological distress by cancer site. Psychooncology 2001;10:19-28. doi: 10.1002/1099-1611(200101/02)10:1<19::AID-PON501>3.0.CO;2-6

9. Faller H, Schuler M, Richard M, Heckl U, Weis J, Ku R. Effects of psycho-oncologic interventions on emotional distress and quality of life in adult patients with cancer: systematic review and meta-analysis. J Clin Oncol 2014;31:782-93. doi: 10.1200/ JCO.2011.40.8922

10. Lam WWT, Shing YT, Bonanno GA, Mancini AD, Fielding R. Distress trajectories at the first year diagnosis of breast cancer in relation to 6 years survivorship. Psychooncology 2012;21:90-9. doi: 10.1002/pon.1876 Distress

11. Giese-davis J, Collie K, Rancourt KMS, Neri E, Kraemer HC, Spiegel D. Decrease in depression symptoms is associated with longer survival in patients with metastatic breast cancer: a secondary analysis. J Clin Oncol 2015;29:413-20. doi: 10.1200/ JCO.2010.28.4455

12. Yee MK, Sereika SM, Bender CM, Brufsky AM, Connolly MC, Rosenzweig MQ. Symptom Incidence, distress, cancer-related distress, and adherence to chemotherapy among african american women with breast cancer. Cancer 2017;123:2061-9. doi: 10.1002/cncr.30575

13. Eakin EG, Strycker LA. Awareness and barries to use of cancer support and information resources by HMO patients with breast, prostate, or colon cancer: Patient and provider perspectives. Psychooncology 2001;10:103-13. doi: 10.1002/pon.500

14. Ministerio de Sanidad y Política Social. Estrategia en Cáncer del Sistema Nacional de Salud [Internet]. Madrid: Ministerio de Sanidad y Política Social, Centro de Publicaciones;2010. [Accesoel1 demarzode2020]. Disponibleen: https://www.mscbs. gob.es/organizacion/sns/planCalidadSNS/pdf/ActualizacionEstrategiaCancer.pdf 
15. Travado L, Reis JC, Watson M, Borràs J. Psychosocial oncology care resources in Europe: a study under the European Partnership for Action Against Cancer (EPAAC). Psychooncology 2017;26:523-30. doi: 10.1002/pon.4044

16. Winn RJ, Mcclure J. The NCCN Clinical Practice guidelines in oncology: A primer for users. J Natl Compr Cancer Netw 2003;1:5-13. doi: 10.6004/jnccn.2003.0003

17. Neuss MN, Desch CE, Mcniff KK, Eisenberg PD, Gesme DH, Jacobson JO, et al. A process for measuring the quality of cancer care: the quality oncology practice initiative. J Clin Oncol 2005;23:6233-9. doi: 10.1200/JCO.2005.05.948

18. Zebrack B, Kayser K, Sundstrom L, Savas SA, Henrickson C, Acquati C, et al. Psychosocial distress screening implementation in cancer care: An analysis of adherence, responsiveness, and acceptability. J Clin Oncol 2015;33:1165-70. doi: 10.1200/JCO.2014.57.4020

19. Mcleod DL, Ph RND, Morck AC. A pan-Canadian web-based education program to support screening for distress: Evaluation of outcomes. Palliat Support Care 2014;12:1523. doi: $10.1017 / \mathrm{S} 1478951513000072$

20. Carlson LE, Waller A, Mitchell AJ. Screening for Distress and Unmet Needs in Patients With Cancer: Review and Recommendations. J Clin Oncol 2015;30:1160-77. doi: 10.1200/JCO.2011.39.5509

21. Baumeister H, Reichler L, Munzinger M, Lin J. The impact of guidance on Internetbased mental health interventions — A systematic review. Internet Interv 2014;1:20515. doi: 10.1016/j.invent.2014.08.003

22. Dieng M, Cust AE, Kasparian NA, Mann GJ, Morton RL. Economic evaluations of psychosocial interventions in cancer: a systematic review. Psychooncology 2016;25:1380-92. doi: 10.1002/pon.4075 Review

23. Holmes MM. Why people living with and beyond cancer use the internet. Integr Cancer Ther 2019;18(1). doi: 10.1177/1534735419829830

24. Nguyen SKA, Ingledew P-A. Tangled in the breast cancer web: an evaluation of the usage of web-based information resources by breast cancer patients. J Cancer Educ 2013;28:662-8. doi: 10.1007/s13187-013-0509-6

25. Ochoa-Arnedo C, Flix-Valle A, Casellas-Grau A, Casanovas-Aljaro N, Herrerro O, Sumalla EC, et al. An exploatory study in breast cancer of factors involved in the use and communication with health professionals of Internet information. Support Care Cancer. 2020;7 February:1-8. doi: 10.1007/s00520-020-05335-x

26. Valero-Aguilera B, Bermúdez-Tamayo C, García-Gutiérrez JF, Jiménez-Pernett J, CózarOlmo JM, Guerrero-Tejada R, et al. Information needs and Internet use in urological and breast cancer patients. Support Care Cancer 2014;22:545-52. doi: 10.1007/s00520-0132009-y

27. Carma L Bylund, Gueguen JA, Agostino TAD, Imes RS, Sonet E. Cancer patients' decisions about discussing Internet information with their doctors. Psychooncology 2009;18:1139-46. doi: 10.1002/pon.1511

28. Eysenbach G. What is e-health? J Med Internet Res 2001;3(2):e:20. doi: 10.2196/ jmir.3.2.e20

29. Luckett T, Butow PN, King MT. Improving patient outcomes through the routine use of patient-reported data in cancer clinics: Future directions. Psychooncology 2009;18:1129-38. doi: 10.1002/pon.1545

30. Berry DL, Hong F, Halpenny B, Partridge AH, Fann JR, Wolpin S, et al. Electronic selfreport assessment for cancer and self-care support: Results of a multicenter randomized trial. J Clin Oncol 2014;32:199-205. doi: 10.1200/JCO.2010.30.3909 
31. Velikova G, Booth L, Smith AB, Brown PM, Lynch P, Brown JM, et al. Measuring quality of life in routine oncology practice improves communication and patient wellbeing: A randomized controlled trial. J Clin Oncol 2004;22:714-24. doi: 10.1200/ JCO.2004.06.078

32. Seow BH, Sussman J, Martelli-Reid L, Pond G, Bainbridge D. Do high symptom scores trigger clinical actions? An audit after implementing electronic symptom screening. J Oncol Pract 2012;8:e142-e148. doi: 10.1200/JOP.2011.000525

33. Kotronoulas G, Kearney N, Maguire R, Harrow A, Domenico D Di, Croy S. What Is the value of the routine use of patient-reported outcome measures toward improvement of patient outcomes, processes of care, and health service outcomes in cancer care? A systematic review of controlled trials. J Clin Oncol 2014;32:1480-501. doi: 10.1200/ JCO.2013.53.5948

34. Berry DL, Blumenstein BA, Halpenny B, Wolpin S, Fann JR, Austin-Seymour M, et al. Enhancing patient-provider communication with the electronic self-report assessment for cancer: A randomized trial. J Clin Oncol 2015;29:1029-35. doi: 10.1200/ JCO.2010.30.3909

35. van den Berg SW, Gielissen MFM, Ottevanger PB, Prins JB. Rationale of the BREAst cancer e-healTH [BREATH] multicentre randomised controlled trial: An Internetbased self-management intervention to foster adjustment after curative breast cancer by decreasing distress and increasing empowerment. BMC Cancer 2012;12:1-13. doi: 10.1186/1471-2407-12-394

36. McAlpine H, Joubert L, Martin-Sanchez F, Merolli M, Drummond KJ. A systematic review of types and efficacy of online interventions for cancer patients. Patient Educ Couns 2015;98:283-95. doi: 10.1016/j.pec.2014.11.002

37. Lleras M, Casellas $\square$ Grau A, Sumalla E, Rodriguez Ortega A, Borràs Andrés JM, Ohoa C. Randomized Control Trial (RCT) of online vs presencial positive group psychotherapy. Psychooncology, Suppl Abstr 2017 World Congr Psycho-Oncology, 14-18 August 2017, Berlin, Ger. 2017;26(S3):44-5. doi: 10.1002/pon.4476

38. National Cancer Institute. Common Terminology Criteria for Adverse Events (CTCAE) 4.0 [Internet]. U.S. Department of Health and Human Services; 2010. [Acceso 1 de marzo de 2020]. Disponible en: https://www.eortc.be/services/doc/ctc/ CTCAE_4.03_2010-06-14_QuickReference_5x7.pdf

39. Institut Català d'Oncologia. [Internet]. 2020. [Acceso 1 de amrzo de 2020]. Disponible en: https://www.psicooncologiaonline.com/

40. Ochoa C, Sumalla EC, Maté J, Castejón V, Rodríguez A, Blanco I, et al. Psicoterapia positiva grupal en cáncer. hacia una atención psicosocial integral del superviviente de cáncer. Psicooncologia. 2010;7:7-34.

41. Ochoa Arnedo C, Casellas-Grau A. Positive Psychotherapy in Cancer: Facilitating Posttraumatic Growth in Assimilation and Accommodation of Traumatic Experience. In: Martin CR, Preedy VR, Patel VD (Eds.) Comprehensive guide to post-traumatic stress disorder. New York: Springer Verlag; 2016. p. 2133-49. doi: 10.1007/978-3-31908613-2_77-1

42. Spiegel D, Classen C. Group therapy for cancer patients: A Research-Based Handbook of Psychosocial Care (1st ed.). New York: Basic Books; 2000.

43. Ochoa-Arnedo C, Casellas $\square$ Grau A, Lleras de Frutos M, Vives J. Stress Control or Posttraumatic Growth facilitation to diminish Distress in Cancer Survivors? A Randomized Control Trial. J Psychosoc Oncol Res Pract 2019;1(1S):95. doi: 10.1097/ OR9.0000000000000010 
44. Ochoa C, Casellas-Grau A, Vives J, Font A, Borràs J-M. Positive psychotherapy for distressed cancer survivors: Posttraumatic growth facilitation reduces posttraumatic stress. Int J Clin Heal Psychol 2017;17:28-37. doi: 10.1016/j.ijchp.2016.09.002

45. Melville KM, Casey LM, Kavanagh DJ. Dropout from internet-based treatment for psychological disorders. Br J Clin Psychol 2010;49:455-71. doi: $10.1348 / 014466509 X 472138$ 